\title{
Influence of seismic isolation systems and soil-structure interaction on the response of structures
}

\section{Influence des systèmes d'isolation parasismique et de l'interaction sol- structure sur la réponse des structures}

Hasrouri Samah ${ }^{1}$ et Ounis Abdelhafid ${ }^{2}$

${ }^{1}$ Laboratoire de Recherche en Hydraulique Appliquée, LRHYA, Université Hadj Lakhder, Batna, Algérie

\begin{abstract}
The reduction of cyclic loading triggering major damage in urban areas is a major challenge in earthquake engineering. The processes of structural control especially control structures for passive isolation systems and earthquake sinks of energy, which consists in superimposing on the structure a device which modifies the rigidity or the damping of the structural system without the demand for an external energy source and without introducing energy for its operation, these devices with taking account the effect of soilstructure interaction are currently regarded as effective solutions to these problem by reducing the level of acceleration imposed on the structure and consequently forces shear and the relative displacements in the superstructure. This reduction of shear forces and displacements will limit the structural damage.
\end{abstract}

\section{Introduction}

L'isolation sismique à la base est une méthode de conception parasismique qui consiste à introduire entre les fondations et la superstructure, des dispositifs qui ont une déformabilité horizontale très importante et une rigidité verticale très élevée. Ces dispositifs permettent à découpler le mouvement du sol de la structure dans le but de réduire les forces transmises à cette dernière. L'isolateur capte les déformations (inélastiques) et filtre les accélérations (hautes fréquences) de sorte que la superstructure isolée se déplace essentiellement selon un mode rigide subissant de faibles accélérations et presque pas de déformations.

Les isolateurs parasismiques en élastomère font partie des types d'isolateur les plus utilisés à cause de leur facilité de fabrication, d'installation et d'entretien. Toutefois, ces isolateurs présentent un comportement linéairement élastique et visqueux à grandes déformations de cisaillement dépendant de plusieurs facteurs qui varient durant la phase d'utilisation (température, déformation interne, fréquence de chargement...).

Il s'ensuit que, pour assurer la sécurité de l'ouvrage isolé, il est important de connaître l'impact de la variation de chacun des facteurs sur les propriétés mécaniques des isolateurs en caoutchouc.
L'objectif de ce travail est d'étudier l'influence des systèmes d'isolation parasismique à base d'élastomères comme moyen de contrôle passif sur la réponse sismique des structures avec tenir compte l'interaction solstructure.

\section{2 procédures de simulation numérique}

\subsection{Présentation du modèle}

Une structure sera modélisée avec et sans prise en compte de l'effet d'interaction sol-structure en considérant l'isolation parasismique à la base de type LRB (lead rubber bearing), et l'analyse de l'influence de ces derniers sur le comportement des structures avec le logiciel SAP2000.

IL s'agit d'un portique en béton armé de $5,0 \mathrm{~m}$ d'ouverture " figure .1". Les poteaux de $3.14 \mathrm{~m}$ de hauteur, ont une section de $0,3 \times 0,4 \mathrm{~m}^{2}$. Le plancher est constitué d'une dalle en corps creux de $16+4 \mathrm{~cm}$ d'épaisseur et dimensions en plan de 4,0×5,0 m. La structure repose sur des semelles superficielles en béton de section carrée de $2 \mathrm{~m}$ de côté. 


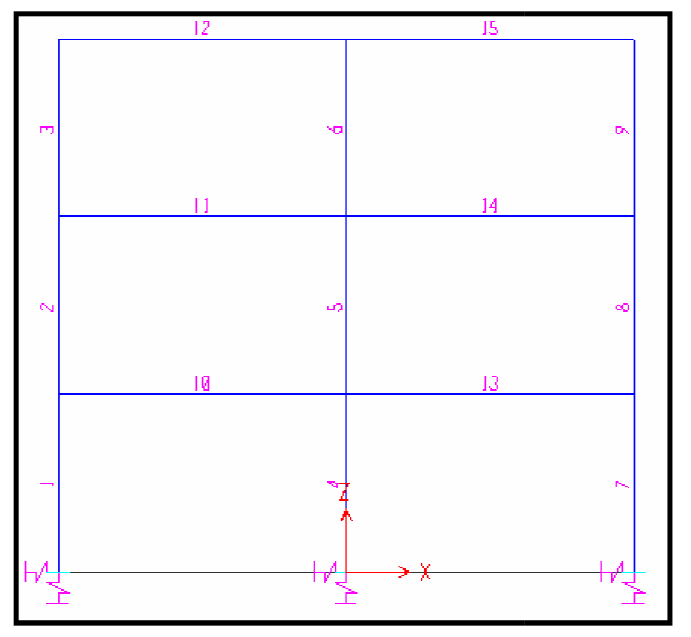

Fig. 1.Portique sur des appuis élastiques

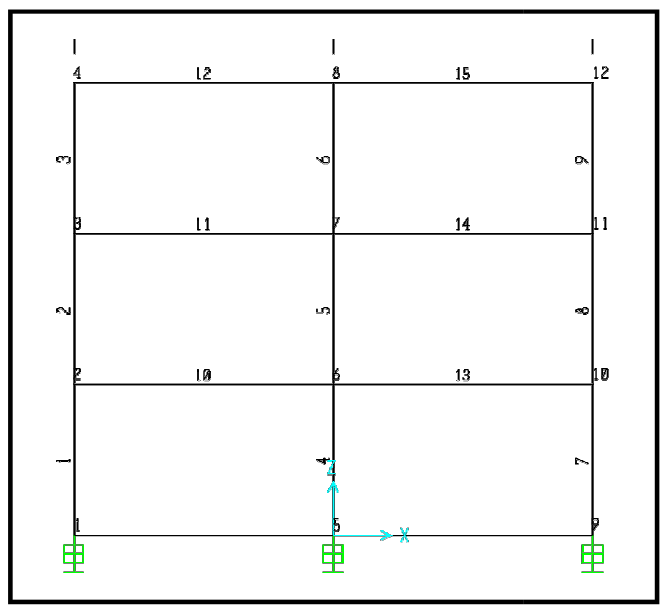

Fig. 2.Portique avec des isolateurs à la base(LRB)

\subsection{Caractéristiques des matériaux}

\subsubsection{Caractéristiques géotechniques du sol}

Pour l'étude de l'interaction sol-structure (ISS) [1]. Le sol est modélisé par un ressort horizontal, un ressort vertical et un ressort de basculement en chaque nœud (Capra et al. 84), (Oudjene et al. 01).

Le modèle ressort de sol " figure .1" définit les réactions élastiques de ce dernier à l'égard des composantes de déplacement statique de la fondation.

Les valeurs des raideurs des ressorts de sol sont calculées à l'aide des relations données dans le tableau 1 .

Les valeurs des coefficients sans dimension $\beta \mathrm{x}, \mathrm{y}, \quad \beta$ $\mathrm{z}$ et $\beta \varphi, \psi$, du tableau 2 sont données par des abaques spécifiques en fonction du rapport des dimensions des semelles et la direction de l'action sismique considérée (Zacek, 96).

Le sol (sable) est supposé homogène avec un comportement élastique linéaire et ses propriétés sont résumées dans le tableau 2 .
Table 1. Raideurs équivalentes pour un milieu semi-infini [1]

\begin{tabular}{|c|c|}
\hline Mouvement & Semelle rigide rectangulaire \\
\hline Horizontal x ou y & $\mathrm{K}_{\mathrm{x}, \mathrm{y}}=\frac{\mathrm{E}}{2\left(1-\mathrm{v}^{2}\right)} \beta_{\mathrm{x}, \mathrm{y}} \sqrt{\mathrm{b} \cdot \mathrm{a}}$ \\
\hline Vertical & $\mathrm{K}_{\mathrm{z}}=\frac{\mathrm{E}}{2\left(1-\mathrm{v}^{2}\right)} \beta_{\mathrm{z}} \sqrt{\mathrm{b} \cdot \mathrm{a}}$ \\
\hline Basculement & $\mathrm{K}_{\Psi, \varphi}=\frac{\mathrm{E}}{2\left(1-\mathrm{v}^{2}\right)} \beta_{\Psi, \varphi} \mathrm{b}^{2} \sqrt{\mathrm{b} \cdot \mathrm{a}}$ \\
\hline
\end{tabular}

Table 2. Caractéristiques des sols

\begin{tabular}{|c|c|c|c|}
\hline \multicolumn{2}{|c|}{ Sites } & $\begin{array}{r}\begin{array}{c}\text { Site meuble } \\
\text { (sable lâche) }\end{array} \\
\end{array}$ & $\begin{array}{l}\text { Site très meuble } \\
\text { (sable très lâche) }\end{array}$ \\
\hline \multicolumn{2}{|c|}{$\begin{array}{c}\text { Module de Young } \\
\text { E(Mpa) }\end{array}$} & 10 & 5 \\
\hline \multicolumn{2}{|c|}{$\begin{array}{c}\text { Coefficient de } \\
\text { poisson } v\end{array}$} & 0.5 & 0.5 \\
\hline \multicolumn{2}{|c|}{$\begin{array}{c}\text { Densité volumique } \\
(\mathrm{KN} / \mathrm{cm} 3\end{array}$} & 17 & 19 \\
\hline \multicolumn{2}{|c|}{$\begin{array}{c}\text { Angle de } \\
\text { frottement (deg) }\end{array}$} & 35 & 35 \\
\hline \multicolumn{2}{|c|}{ Cohésion (KN/M2) } & 0 & 0 \\
\hline \multirow{3}{*}{$\begin{array}{c}\text { Paramètre } \\
\text { sans } \\
\text { dimension }\end{array}$} & $\beta x, y$ & 1.42 & 1.42 \\
\hline & $\mathrm{Bz}$ & 2.12 & 2.12 \\
\hline & $\beta \psi, \varphi$ & 1.65 & 1.65 \\
\hline \multirow{3}{*}{$\begin{array}{c}\text { raideurs } \\
\text { des } \\
\text { ressorts de } \\
\text { sol }(\mathbf{k n} / \mathbf{m}) \\
\end{array}$} & $\mathbf{K x}, \mathbf{y}$ & 18930 & 11833.33 \\
\hline & $\mathbf{K z}$ & 28260 & 17666.66 \\
\hline & $\mathbf{\psi} \psi, \varphi$ & 88000 & 85937.5 \\
\hline
\end{tabular}

\subsubsection{Caractéristiques de l'isolateur $L R B$}

Les isolateurs à base d'élastomère " figure .2", modélisés par des éléments Link, ont été fournis à tous les supports des poteaux à la base. Les propriétés des Link ont été fournis par des calculs du modèle d'isolateur de base LRB.

Les calcule détaillé de l'isolateur de base " figure .3 et 4 " ont été obtenus par les codes IBC2000 et IBC2006 pour finaliser la conception des isolateurs de base.

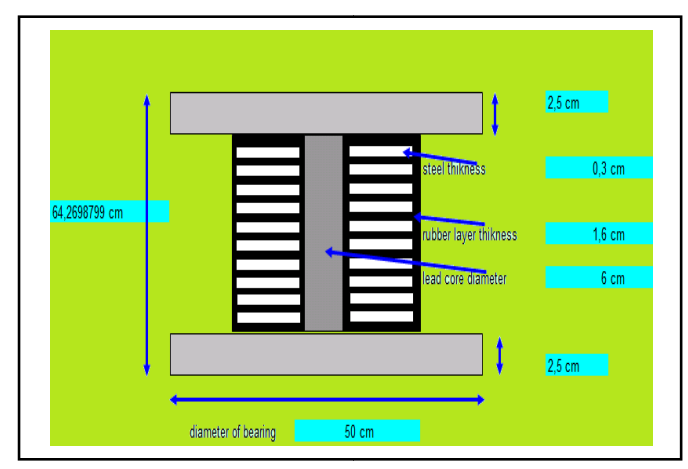

Fig. 3. Les isolateurs utilisés à base d'élastomère (Site meuble) 


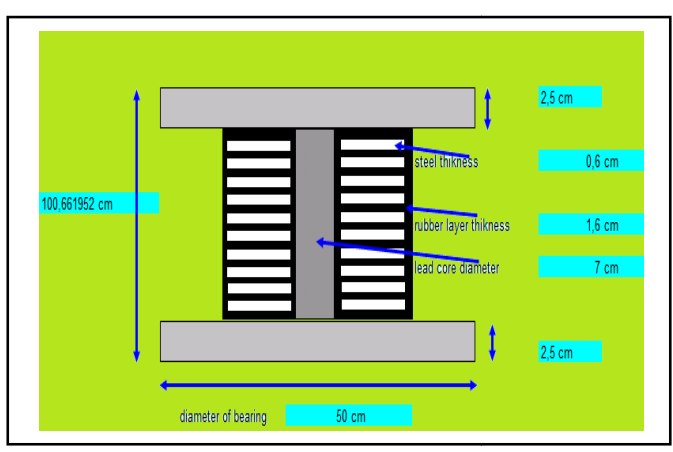

Fig. 4. Les isolateurs utilisés à base d'élastomère (Site très meuble)

Pour une analyse linéaire modale " figure .5"

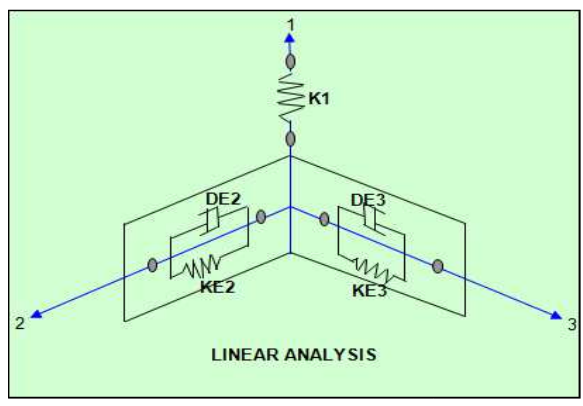

Fig. 5. Modèle du système d'isolation LRB pour une analyse linéaire

Le modèle utilisé pour caractériser le comportement hystérèse du LRB " figure .6" est exigé généralement la définition de trois paramètres à savoir :

La rigidité post-élastique $\mathrm{Kp}$, la force élastique Fy et le déplacement élastique Dy [6].

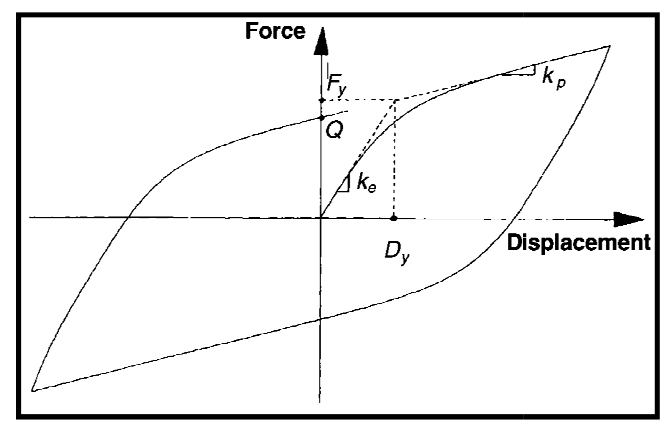

Fig. 6. Model Relation idéale hystérèse force-déplacement d'un appui en caoutchouc de plomb [2]

-La rigidité effective $K e f f=K p+\frac{Q}{D} \quad D \geq D_{y}$

-La rigidité élastique est approximativement égale à 6,5 à

$10 \mathrm{Kp}$

-La période naturelle

$T=2 \Pi \sqrt{\frac{W}{K_{\text {eff }} g}}$

-Le taux effectif d'amortissement effectif

$B e f f=\frac{4 Q(D-Q / 9 K p)}{2 \Pi(K p D+Q) D}$

-Le déplacement au centre de rigidité

$D=\left(\frac{g}{4 \Pi 2}\right) \frac{S_{d 1} T_{d}}{B_{d}}$
$S_{d 1}=\frac{2}{3} S M 1$

Où : g est l'accélération,

Sd1 est le coefficient spectral,

Td est la période isole,

Bd est le coefficient d'amortissement.

Et

Où

$$
S_{M 1}=F v S_{1}
$$

- S1 : est 5\% d'amortissement d'accélération spectrale pour le site disponible sur les cartes accompagnant à IBC2000.

- Fv : le coefficient de site défini pour les classes des divers sites et des niveaux d'accélération.

Les caractéristiques d'isolateur LRB $[9,10,11]$ sont données dans le tableau 3.

Table 3. Caractéristiques d'isolateur LRB

\begin{tabular}{|c|l|l|}
\hline \multirow{2}{*}{ Classe de site } & Site meuble & \multicolumn{1}{|c|}{$\begin{array}{c}\text { Site très } \\
\text { meuble }\end{array}$} \\
\cline { 2 - 3 } & $\mathrm{D}$ & $\mathrm{E}$ \\
\hline $\begin{array}{c}\text { Coefficient } \\
\text { spectral Sd }\end{array}$ & 0.56 & 0.89 \\
\hline $\begin{array}{c}\text { K1=Kv } \\
\mathbf{( k N / m 2 )}\end{array}$ & 687637,21 & 432670,61 \\
\hline $\begin{array}{c}\text { KE2 } \\
(\mathbf{k N} / \mathbf{m} 2)\end{array}$ & 350,86 & 350,45 \\
\hline $\mathbf{D E 2}$ & 0,0945 & 0,094 \\
\hline
\end{tabular}

\section{Résultats}

\subsection{Effet de l'interaction sol structure (ISS) et d'isolations parasismiques à la base (LRB) sur la valeur de l'effort tranchant à la base}

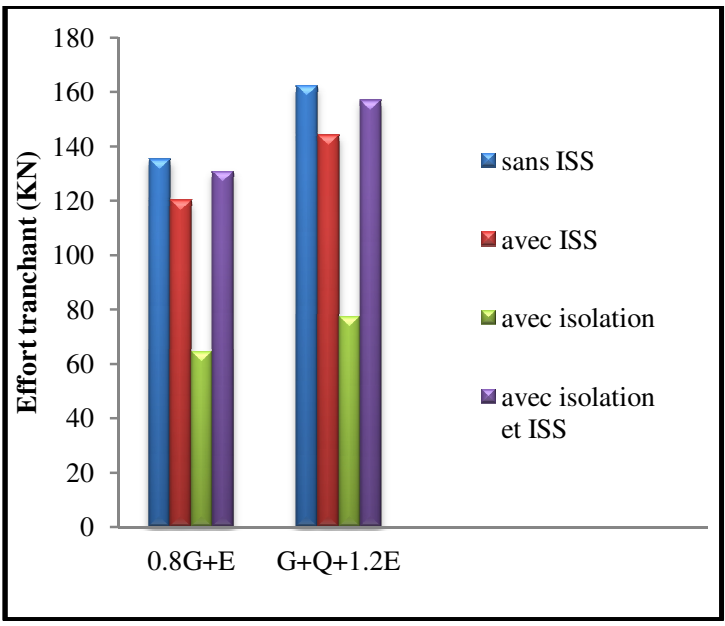

Fig. 7. Effort tranchant à la base sans et avec isolation et ISS des sites meubles 


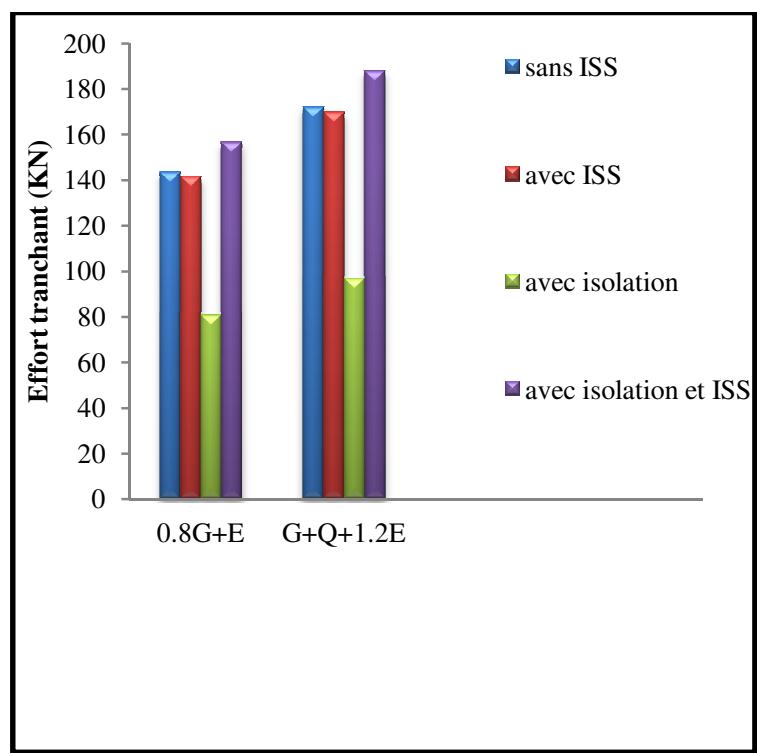

Fig. 8. Effort tranchant a la base sans et avec isolation et ISS des sites très meubles

\subsection{Effet d'isolation parasismique et ISS sur les} déplacements horizontaux des nœuds

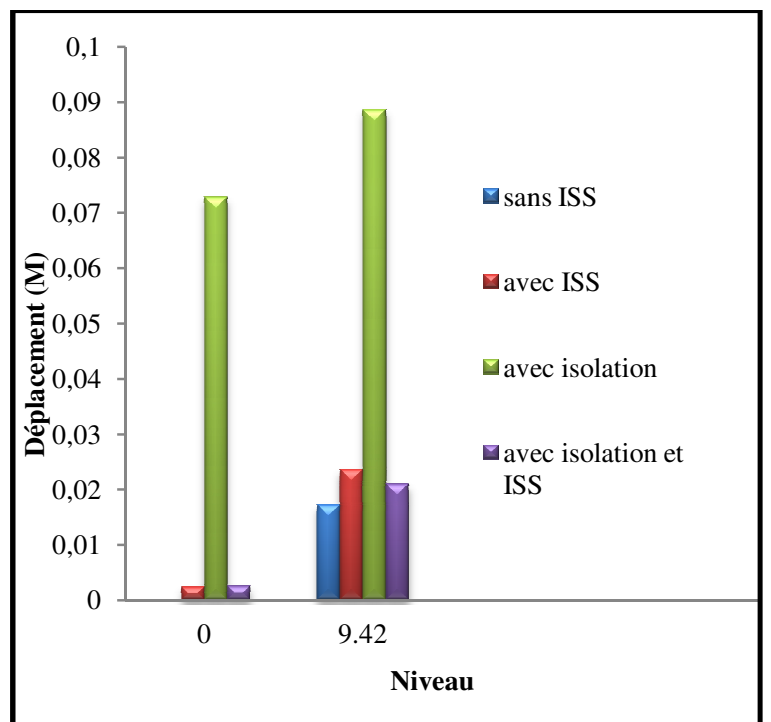

Fig. 9. Déplacements horizontaux avec isolation et ISS des sites meubles

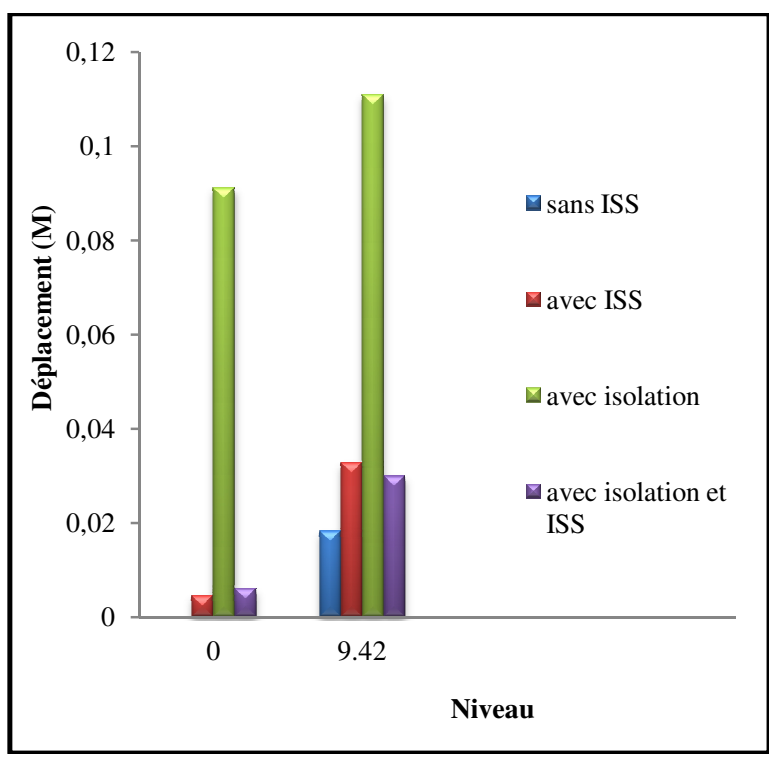

Fig. 10. Déplacements horizontaux avec isolation et ISS des sites très meubles

\subsection{Effet d'isolation parasismique et ISS sur les déplacements verticaux des nœuds}

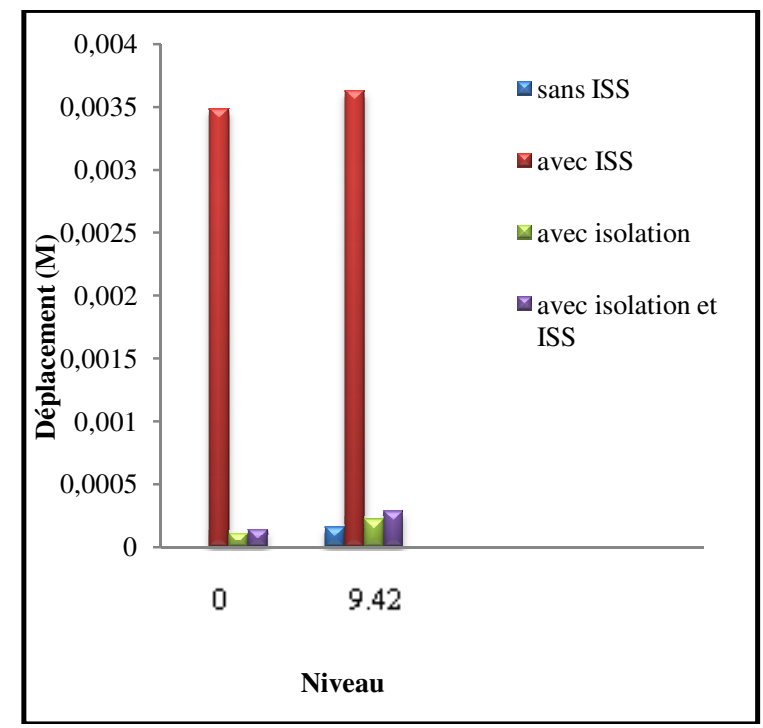

Fig. 11. Déplacements verticaux avec isolation et ISS des sites meubles 


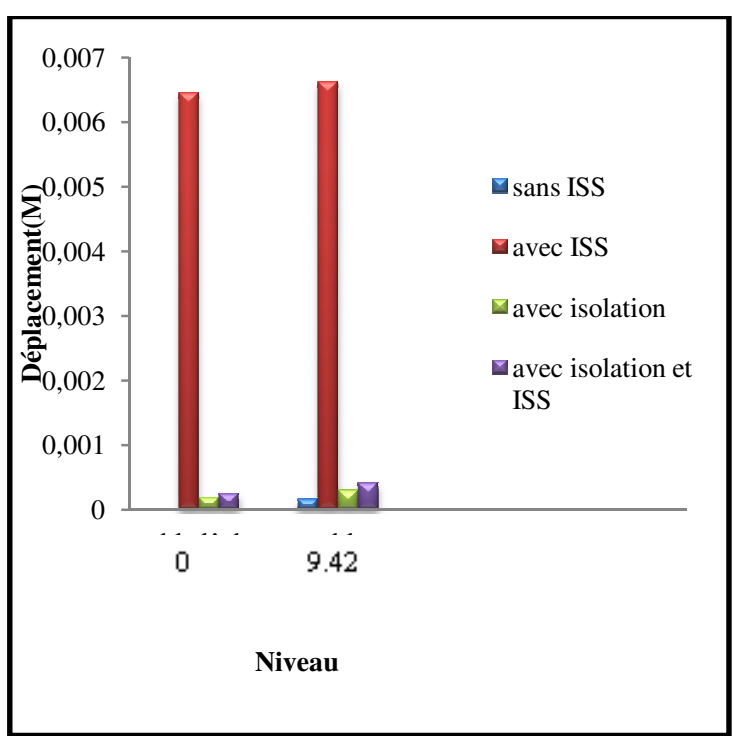

Fig. 12. Déplacements verticaux avec isolation et ISS des sites très meubles

\section{Conclusion}

Les conclusions principales tirées de la présente étude sont :

-La modélisation des structures avec prise en compte de l'effet ISS est le plus proche de la réalité car sa dépendance des caractéristiques des sols est plus importante.

-La modélisation des structures encastrées (sans tenir compte l'effet ISS) n'est pas à recommander car elle donne des résultats trop conservatifs.

-Les systèmes d'isolation parasismique (appui en caoutchouc à faible- amortisseurs avec un noyau de plomb (LRB)), peuvent être un moyen efficace pour limiter les effets dévastateurs d'un séisme sur une structure donnée

-les efforts tranchants de la structure munie de systèmes d'isolation et avec prise en compte l'effet ISS sont supérieurs à ceux de la structure soumise à l'effet d'interaction. Cependant les déplacements prennent des valeurs inferieurs.

-Les déplacements horizontaux des structures munies des systèmes d'isolation et soumis à l'effet d'interaction sont inférieurs à ceux des structures avec l'effet d'interaction.

-Les déplacements verticaux des structures et des fondations munies des systèmes d'isolation et soumis à l'effet d'interaction sont inférieurs à ceux des structures avec l'effet d'interaction sol structure.

-Les structures munies des systèmes d'isolation et soumise à l'effet d'interaction limiteront les dommages structurales des sites lâches et très lâches par la réduction des efforts de cisaillement et des déplacements.

Il est nécessaire de tenir compte de certains aspects actuellement non pris en compte dans le RPA 2003 :

1-Les règles RPA20003 doivent donner plus de détails concernant les différents aspects inhérents à une analyse de l'influence de l'interaction sol structure.

2-Les règles RPA20003 doivent donner des études spécifier concernant les différents systèmes d'isolation parasismique et pour l'utilisation de ces derniers doivent être adoptées.

3-Spécifier le type de structures pour lesquelles l'interaction sol-structure est importante et donner des directives pour les calculs.

4-le règlement algérien stipule qu'en cas de non disponibilité d'étude de sol, un site 3 sera pris en compte dans l'étude sismique, il est noté que cette considération conduit à un surdimensionnement coûteux plus que les frais qui seraient engagés pour une étude de sol. De ce fait il faudrait insister plus sur bonne étude de sol qui permet une économie et une sécurité.

\section{Références}

1. Alain Pecker .Comportement des sols interaction sol-structure.

2. Anil K. Chopra University of Califomia at Berkeley, dynamics of structures theory and applications to Earthquake Engineering.

3. Davidovici V. La construction en zone sismique, Paris, Editions Le Moniteur (1999).

4. Michael D. Symans, $\mathrm{PhD}$, seismic protective systems passive energy dissipation.

5. Trevor E Kelly, S.E.Holmes Consulting Group Ltd Revision 0 (July 2001 DESIGN GUIDELINES).

6. F Naeim-Design of structures with seismic isolation.

7. A.Pecket, Dynamique des sols (Presse de l'école des nationales des ponts et Chausses 1984).

8. KHALIL Louay, SADEK Marwan \& SHAHROUR Isam Influence de l'interaction solstructure (ISS) sur la fréquence fondamentale.

9. (2006) INTERNATIONAL BUILDING CODE®

10. (2000)IBC

11. Paul W Meisel Member VISCMA, Understanding the (2000) IBC Code (Architectural Components and Equipment Restraint)

12. KINETICS ${ }^{\mathrm{TM}}$ Guide to Understanding IBC Seismic for $M E P$, required basic project information

13. Règle parasismiques algériennes (RPA99/version 2003), Ministère de l'habitat Centre national de recherché appliqué en génie parasismique. 